\title{
From Field Data to Attitude Formation
}

\author{
Kei-Léo Brousmiche ${ }^{1,4}$, Jean-Daniel Kant ${ }^{1}$, Nicolas Sabouret ${ }^{2}$, François \\ Prenot-Guinard $^{4}$, Stéphane Fournier ${ }^{4}$ \\ 1 LIP6 - CNRS UMR 7606, \\ Université Pierre et Marie Curie, France kei-leo.brousmiche@lip6.fr \\ jean-daniel.kant@lip6.fr \\ 2 LIMSI-CNRS, UPR 3251, \\ Université Paris-Sud, France nicolas.sabouret@limsi.fr \\ 3 Airbus Defense \& Space \\ 1 bd Jean-Moulin, Elancourt, France francois.prenot-guinard@airbus.com \\ stephane.fournier@airbus.com
}

\begin{abstract}
This paper presents a multi-agent model for simulating attitude formation and change based on perception and communication in the context of stabilization operations. The originality of our model comes from (1) attitude computation that evaluates information as part of a history relative to the individual (2) a notion of co-responsibility for attitude attribution. We present a military scenario of French operations in Afghanistan along with polls results about the opinion of citizen toward present Forces. Based on these field data, we calibrate the model and show the resulting attitude dynamics. We study the sensibility of the model to the co-responsibility factor.
\end{abstract}

\section{Introduction}

The new conflicts that arouse during the two last decades have brought a deep shift in military strategies [19]: most of the stabilization operations conducted by western forces involves opponents who blend themselves into the "human environment" to turn the population in their favor. In order to counter them, one should not only rely on tactical actions against them but also on non-kinetic actions such as reconstruction or specific communication actions that aim at altering the "hearts and minds" of the population. In this context, understanding the impact of actions performed by the intervention Force on the population's attitude is a major issue.

The concept of attitude derives from social psychology and could be defined as "a mental and neural state of readiness organized through experience" [2]. Multi agent simulation of attitude dynamics seems a promising approach to study such complex social phenomenon since it is funded on individuals micro modeling and their interactions to analyze emergent macro trends [7]. While multiple agentbased models have been proposed to study attitude and opinion (i.e. expressed attitude) dynamics $[5,20,3]$, the major difficulty relies in validation: can a given model correctly reflect the attitude dynamics of a population in a conflict zone? 
Opinion polls can provide target values. However, collecting field data to feed the simulation model and assessing the validity of its outcome (based on the expected values) can prove to be very difficult.

As part of our research, we have been given an access to polls results about opinions of the population toward the different present Forces (foreign Force and Taliban) in an area of Afghanistan where French forces conducted stabilization operations. These results have been collected by Altai Consulting for the French Ministry of Defense ${ }^{4}$. Our analysis of these data has brought light to attitude dynamics that go against classical approaches in social simulation. First, most models compute the attitude as the aggregation of the impact of each feature, seen as independent criteria or events $[20,5]$. However, people do not evaluate each action (such as food provision, military patrol, bombing attack, etc.) independently but towards what similar actions represents in general in terms of direct and indirect consequences for the population. It corresponds to Kahneman's memory-based, retrospective approach of evaluation [14]. Second, we noticed that population's attitude toward the UN army could decrease when it fails to accomplish its securing mission. For instance, in case of a bombing attack, victims will not only blame insurgents who are directly responsible but also the Security Force which have "failed" to prevent such an event. It corresponds in this case to the concept of "role-responsibility" in the sense of Hart [11]. More generally, people tend to attribute the responsibility of an action to other people, groups or situational factors in addition to the direct responsible $[15,13]$.

In this paper, we propose a multi-agent simulation model based on field data that will help to better comprehend attitude dynamics in conflict zones where the population is confronted to antagonists Forces. The next section presents the related works in social simulation of attitude dynamics to situate our work. Section 3 presents our datasets on the events and opinion polls in Afghanistan. Section 4 describes our model of agent and attitude dynamics. Finally, section 5 shows experimental results through a model calibration based on field data and attitude dynamics analysis.

\section{Related works}

Several researches have already proposed computational models of attitudes, from simple binary approaches [17] to more complex ones (e.g. [12]). However, as was pointed out by [4], most of these models' focuses are limited to the interactions between individuals: they do not consider the construction mechanism of the attitude itself at a cognitive level. On this matter, Urbig and Malitz [20] proposed to represent attitudes as the sum of the evaluations of the object's features that can be seen as beliefs on the object, so as to take into account the cognitive aspect. While this model constitutes an interesting view on attitude formation, it has two limits with respect to our objectives.

First, the attitudes' values are not connected to the beliefs of each agent

\footnotetext{
$\overline{4}$ www.altaiconsulting.com
} 
constituting their personal history. Indeed, their attitude revision mechanism is based on the bounded confidence model (e.g. [5]): when two individuals have attitude values close to each other, agents converge their attitudes. However, it could be possible to combine this with Fazio's model of attitude [9]. This model connects the attitude to its forming beliefs as a set of memory associations. Each of these evaluations is weighted by an accessibility value determining the evaluation's degree of reminiscence. By essence, this model maintains a balance between the cognitive representation of the object and its corresponding attitude.

Second, the attitude model of Urbig and Malitz does not embody an emotional component, while social psychologists have defined the attitude as embodying rational and affective components $[18,9]$. This is the reason why Brousmiche and al. [3] attempted to combine Fazio's model with the Simplicity Theory proposed by Dessalles [6]. However, their model does not consider (1) the aggregation of beliefs into a personal history as proposed by Fazio and (2) the notion of role responsibility impacting attitudes toward other actors than perpetrators of an action.

In this paper, we will take the model presented in [3] as a starting point and extend it in order to take into account these two concepts by adding a mechanism of co-responsibility and modifying the aggregation method of the attitude computation.

\section{Field data}

Scenario In the course of the NATO intervention in Afghanistan to stabilize the country, the French Forces were tasked to maintain security in the regions of Kapisa and Surobi between 2008 and 2012. It is in this context that members of CIAE $^{5}$ were sent in the area from October 2010 to September 2012. As part of a more comprehensive maneuver, their actions were meant to complement conventional security operations by positively influencing the population and its key individuals through communication, reconstruction and humanitarian actions. Through a set of six interviews with all the successive officers in charge (3 colonels and 3 commanders) from the Joint Command for Human Terrain Actions, we managed to rebuild the sequence of the events that took place during their tenures, originating both from the NATO and from the Taliban insurgents. This sequence takes the shape of a scenario (see figure 1).

Each action is characterized by a reach, a frequency and a payoff: how many people were directly affected by the action, how many times per week if it is frequent, how each individual is impacted. These values were defined based on subjective assessments of domain's subject matter experts (including members of CIAE). For instance, we defined the action "patrol" as being triggered by the Blue Force, affecting the population with a positive impact of 30 and a reach of 20 people, repeated 3 times per week in average. Similarly, "IED" (Improvised

\footnotetext{
${ }^{5}$ Joint Command for Human Terrain Actions: in charge of civil-military actions (e.g. reconstruction) and community outreach actions (i.e. attitude influence operations)
} 


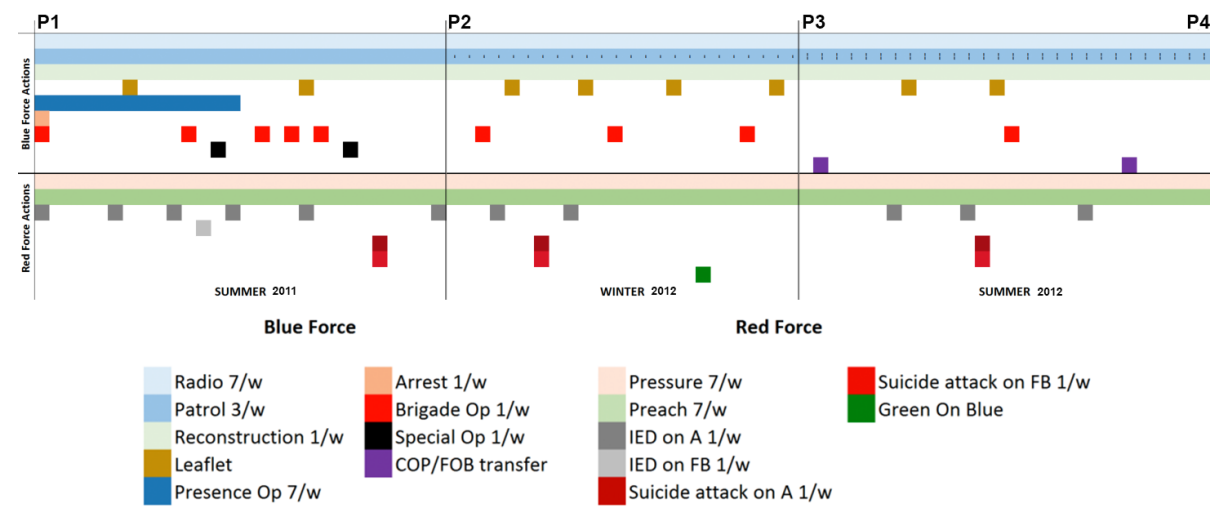

Fig. 1: Scenario of Blue and Red Forces actions. The time points P1, $\cdots$, P4 correspond to the dates of the opinion polls presented in the next section.

Explosive Devices) are done by Red Forces and affect one individual with a payoff of -100 . The number of victims of each attack were defined according to open source records (e.g. [8]).

We can observe that both Forces have a constant background activity toward the population composed of non-kinetic actions. However, the Red Force activity is heavily decreased during winter which corresponds to the second period on the scenario. One reason is that local Taliban leaders leave the region to avoid the arid climate. Also, the surveillance systems, including drones, are more effective in the absence of foliage, making it more difficult for insurgents to place discretely the IEDs. On the Blue Force side, the activity decreases constantly due to the political decision taken after the big human losses on the first period. Indeed, the French government began to adopt a "retreat" strategy after the suicide attack of Joybar (July 13th 2011) which caused considerable human casualties among the Blue Forces.

Opinion polls In order to follow the progress of population's sentiments and to link them to foreign Forces activities, the French Ministry of Defense financed opinion polls in Afghan regions where the French forces were operating. Those surveys were conducted by Afghan contractors between February 2011 and September 2012 with an interval of approximately 6 months issuing into four measure points P1, P2, P3 and P4 of the opinion of the population of Kapisa

\begin{tabular}{|l|l|l|l|l|l|}
\hline Polls dates & P1 & P2 & P3 & P4 \\
\hline "The Blue Force contributes to security" & 40 & 32 & 24 & 19 \\
\hline "The Red Force is the principal vector of insecurity" & 27 & 60 & 27 & 37 \\
\hline
\end{tabular}

Fig. 2: $\%$ of the population favorable with two questions at different dates 
toward (1) the Blue Force and (2) the Red Force on the period corresponding to our scenario.

Opinions toward Red Force in the context of security decrease in summer periods. This could be explained by their high activity level as exposed in the previous section. As for the Blue Force, the global opinion value keeps decreasing along with their decreasing activities. In overall, the opinion dynamics showed by these polls results are consistent with the scenario previously established.

\section{Model}

\subsection{General approach}

Our model is based on the following principle: a simulation corresponds to the execution of actions (e.g. food distribution, construction of a bridge, bombing attack etc.) by actors (e.g. UN Force, terrorists or others) on a population. Individuals communicate about these actions with the others and form an attitude toward the actors.

In our model, we consider a set of actors $A$ and a set of individuals Ind. Actors represent Forces that act in the simulation and for which we want to analyze the attitudes evolution. Each of them corresponds to a computational automaton executing its actions list specified by the above scenario. Each individual $i \in$ Ind is represented by a computational agent and is characterized by its belief base recording facts he is aware of and his connections to other individuals. For each $i \in$ Ind and actor $\in A$, we denote att $(i$, actor $) \in \mathbb{R}$ the attitude of the individual $i$ toward the actor actor.

Beliefs about actions will be the core element in our model: attitudes and communications will be based on these beliefs. We note $a(i)$ the belief of individual $i$ about an action $a$.

Each $a(i)$ is a tuple: $\langle$ name $(a), \operatorname{actor}(a), \operatorname{coResp}(a), \operatorname{bnf}(a), \operatorname{payof} f(a), \operatorname{date}(a)\rangle$ with:

- name the unique name of the action (e.g. "patrol", "suicide attack")

- actor $\in A$ the actor who performed the action (Blue or Red Force)

- coResp $\in A$ the co-responsible actor of the action, if any (e.g. Blue Force will be co-responsible of "suicide attacks" performed by Red Force)

- bnf $\in$ Ind $\cup A$ the beneficiary of the action, i.e. the individual or actor which undergoes the action

- payoff $\in \mathbb{R}$ the impact value of the action, negative when the action is harmful (e.g. attack) and positive when it is beneficial (e.g. food provision).

- date $\in \mathbb{N}$ the occurrence date of the action.

Attitudes are computed as the aggregation of evaluation of similar actions seized by the individual in his past. Two actions are similar if and only if they have the same name and actor. For instance, two distinct patrols done by the Blue Force in the same area are considered as similar. We call general action the meta action that includes similar actions. We denote $g a(i, a)$ the general action 
corresponding to the action $a$ according to the individual $i$ and $g a(i)$ the list of all general actions that $i$ knows.

Actions can be perceived via direct perception (the agent is beneficiary of the action), actor's communication toward the population (the agent receives a message from the actors) or intra-population communication (the agent receives a message from another individual).

\subsection{Attitude computation}

When an agent receives a new information about an action $a$, it adds it to its belief base (if the action is not already present) and, possibly, communicates about it. Moreover, the agent revises its attitude toward the actor of the action. Our model of attitude construction is based on the model proposed by Fazio [9] (see section 2). In short, Fazio proposes to compute the attitude as the average of beliefs' evaluations (i.e. how much this fact is beneficial) weighted by their accessibilities (i.e. how accessible is the information in the subject's mind).

First we compute the interest of an information to estimate its accessibility and its narrative interest (whether or not to communicate the action to other agents). Second, we evaluate the action based on its payoff. Third, we compute the impact of co-responsibility, if required. Finally, we aggregate these evaluations, weighted by their accessibilities, to compute the attitude.

Interest of an action In order to determine what to base their attitude on and what to communicate to other individuals, agents estimate a model of interest of the actions in their belief base. Following [3], our model of interest is based on the Simplicity Theory of Dessalles [6] which proposes to define the narrative interest $N I$ of an information according to the emotion $E$ and the surprise level $S$ it causes to the individual using the following formula: $N I(a)=2^{\alpha E(a)+(1-\alpha) S(a)}$ where $E$ corresponds to the personal emotional response intensity of the individual when faced to an information and the surprise level $S$ translates the sentiment of unexpectedness felt by the individual. The parameter $\alpha \in[0,1]$ balances these two parts.

The emotional intensity $E$ corresponds to the emotional amplitude experienced by the individual when exposed to an event and follows a logarithmic law in conformity with Weber-Fechner's law of the stimuli. In our case, the stimuli corresponds to actions' payoff. A parameter of personal sensibility $\xi \in[0,1]$ modulates the response intensity.

The surprise $S$ experienced by an individual when exposed to an event derives from a level of raw unexpectedness (e.g. "It is surprising that a Taliban saves a citizen"). This level is reduced by a personal reference of unexpectedness based on a personal experience (e.g. "But I have once been saved by a Taliban before").

While $N I(a)$ corresponds to the narrative interest used as a communication heuristic, we also compute the information's interest corresponding to the accessibility of the information:

$$
\operatorname{interest}(a)=\log (N I(a))=\alpha E(a)+(1-\alpha) S(a)
$$


Action evaluation Fishbein and Ajzen [1] advance that the evaluation of an action is weighted by the attitude toward its beneficiary. For instance, a beneficial action for my enemy is harmful for me. Therefore, we define the evaluation of an action belief as:

$$
\operatorname{evaluation}(a)=\operatorname{payof} f(a) \times \operatorname{att}(i, \operatorname{bn} f(a))
$$

Co-responsibility In our case study, the Blue Force endorses the role of security guardian, thus they are co-responsible of all actions compromising the security of the population including Talibans' attacks from population's perspective. Thus, we introduce a co-responsibility mechanism that enables individual to attribute a fraction $\rho \in[0,1]$, parameter of the simulation, of an action payoff to the co-responsible. This mechanism occurs when an individual faces an action $a$ in which (1) there is a co-responsible actor, (2) its impact is negative (i.e. there is no co-responsibility for beneficial actions) and (3) its evaluation is negative. In that specific case, the individual adds a belief $a^{\prime}$ with actor $(a)=\operatorname{coResp}(a)$ and evaluation $\left(a^{\prime}\right)=\rho \times$ evaluation $(a)$.

Aggregation Let gaList(i,actor) be the list containing all the general actions performed by the actor in the belief base of agent $i$. The attitude att(i,actor) of the individual $i$ toward the actor is given at each time of the simulation by:

$\operatorname{att}(i$, actor $)=\sum_{g a(i) \in g a \text { List }(i, a c t o r)}\left(\sum_{a(i) \in g a(i, a)}\left(\frac{\text { evaluation }(a) \times \operatorname{interest}(a, i))}{|g a(i, a)|}\right)\right)$

\section{Experiments}

In this section, we present the experimental results of our model. We aim to reproduce the results of opinion polls collected on the field using the established scenario of events that took place in Kapisa between September 2011 and September 2013. Since the polls did not asked directly the opinion toward the Red Force but "whether they represent a threat" (see section 3), we decided to take the opposite of these results as the target attitude values.

Simulation settings and initialization We input the action sequence presented in the scenario of both Red and Blue Forces into the simulation scheduler; one tick corresponds to one day: the simulation covers the period between the first and last opinion polls in 554 ticks. The two agents corresponding to each Force will then operate their actions according to the scenario. The artificial population representing the inhabitants of Kapisa is composed of 150 agents connected by an interaction network based on a small-world topology [16] with a degree of 4 (i.e. each individual has 4 neighbors in average). 
Before running the actual simulation, we initialize the population with a personal history for each individual and an attitude corresponding to the value given by P1. Indeed, one of our model originality resides in the fact that the attitude depends on the agent's cognitive state characterized by its beliefs and accessibility values. Thus, we must give individuals an initialization belief with a certain reach and payoff for both attitudes toward Red and Blue Forces. These beliefs represent the interactions with Forces preceding to the simulation span. Another subtle point in our model is that individuals are surprised when they witness a totally new action, resulting in an overestimation of the action's impact. In order to habituate them to certain regular actions (such as patrols, preaches, radio broadcasts etc.) we need to run an initialization scenario before the actual one in which the population is confronted to these actions, until we reach a stable point (approximately 200 ticks).

Calibration method Once the simulation is properly initialized, we calibrate the model parameters using each opinion polls results as objectives. We have four points to calibrate per Force, thus totaling 8 points of calibration. The model parameters are shared among all individuals of the population:

$-\alpha$ the weight of emotional sensibility toward the surprise factor

$-\xi$ the level of sensibility to a stimuli (i.e. payoff)

$-\rho$ the co-responsibility factor of Blue Forces for harmful Red actions

We also have to determine the parameters of initialization actions to attain the first point P1: one positive and one negative action per Force. To do so, we fix their payoff values (negative for the harmful action and positive for the other) and calibrate their reaches.

We define our fitness function as the sum of differences' squares between each point of the opinion poll results and its corresponding percentage of favorable individuals in the simulation. We choose to minimize this fitness using the evolutionary algorithm CMA-ES that is one of the most powerful calibration method to solve this kind of problem [10]. Once the fitness stops progressing over 500 iterations, we interrupt the calibration process and save the parameters. Each calibration iteration is based on the average output on over 20 simulations replica since the model is stochastic.

Calibration results The figure 3 shows the results of our model once its parameters have been calibrated. Plain curves represent the objectives to reach that are based on the collected opinion polls results (see section 3 ) ; dashed curves correspond to the simulation results, with $\alpha=0.70, \xi=0.08$ and $\rho=0.15$ (as obtained by the calibration).

We can observe that the attitude dynamics tendencies are well reproduced. The average difference between results and objective points is $13.25 \%$ with a maximum of $19 \%$ for the last point. This gap between survey and simulation results could be explained by several factors in addition to the model itself: field data are generally inaccurate and capture only a limited part of reality. 


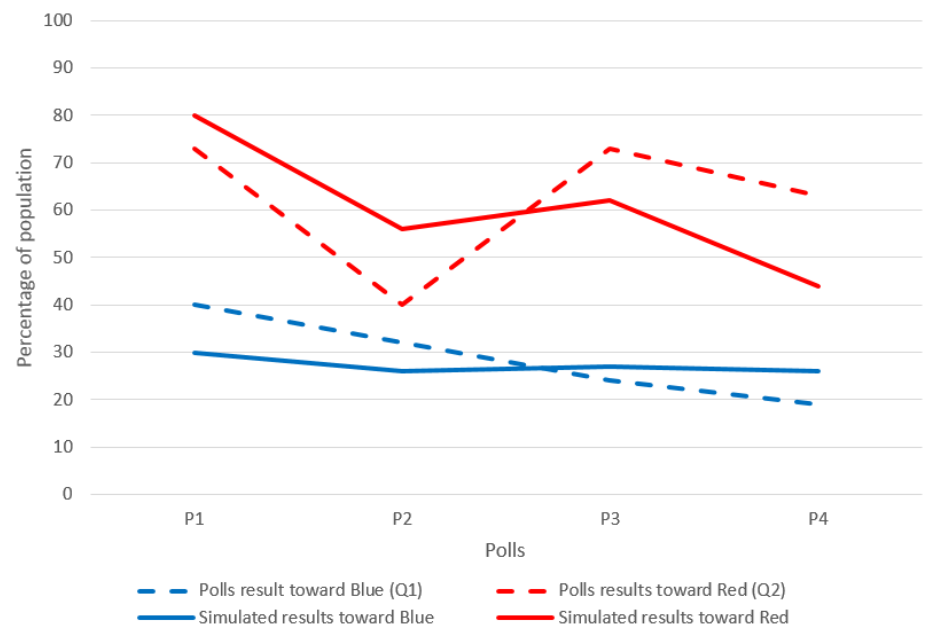

Fig. 3: Simulation results compared to opinion polls

First, the established scenario is based on subjective assessments of some Blue Force officers and do not capture all the military events that took place on the terrain. Adding to this, the parameters of action's models (i.e. payoffs, frequency and reach) have been assessed based on qualitative appraisal of subject matter experts since there is no scientific method to assess them.

Second, the sampling of the opinion survey could not be maintained through the survey process, due to the dynamics of the conflict : certain villages could not be accessed constantly over time due to their dangerousness. Moreover, as it was pointed earlier, the questionnaire did not directly ask the opinion toward Red Force which might increase the gap between our model outputs and the polls results.

Finally, our field data is limited to the context of military events. Even if our study concerns attitudes toward Forces in the military/security context, other events might also have influenced these attitudes such as economic or daily activities.

In view of these limitations, the reproduction of the general tendencies of attitude dynamics between each polls seems encouraging. Besides, these results have been obtained by calibrating only 3 model parameters.

Attitude Dynamics Agent based simulation enables not only to reproduce aggregated data but also to analyze micro behaviors. Figures 4 and 5 below show the dynamics of population's attitudes means values between two polls along with their corresponding scenario.

The decreasing general tendency of the attitude toward the Red Force between P1 and P2 in figure 4 is due to the constant pressure activity that affects negatively the population. We can observe repercussions of each occurrence of action on the attitude dynamics. For instance, in figure 4 we can clearly see 


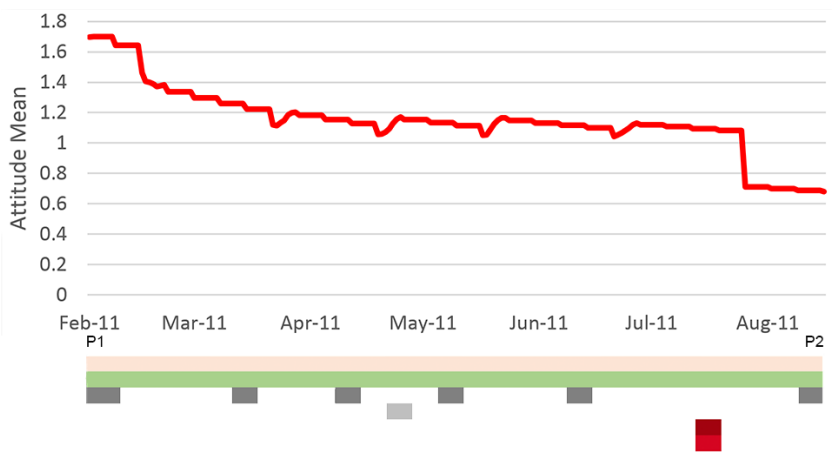

Fig. 4: Attitude mean toward Red Force between P1 and P2

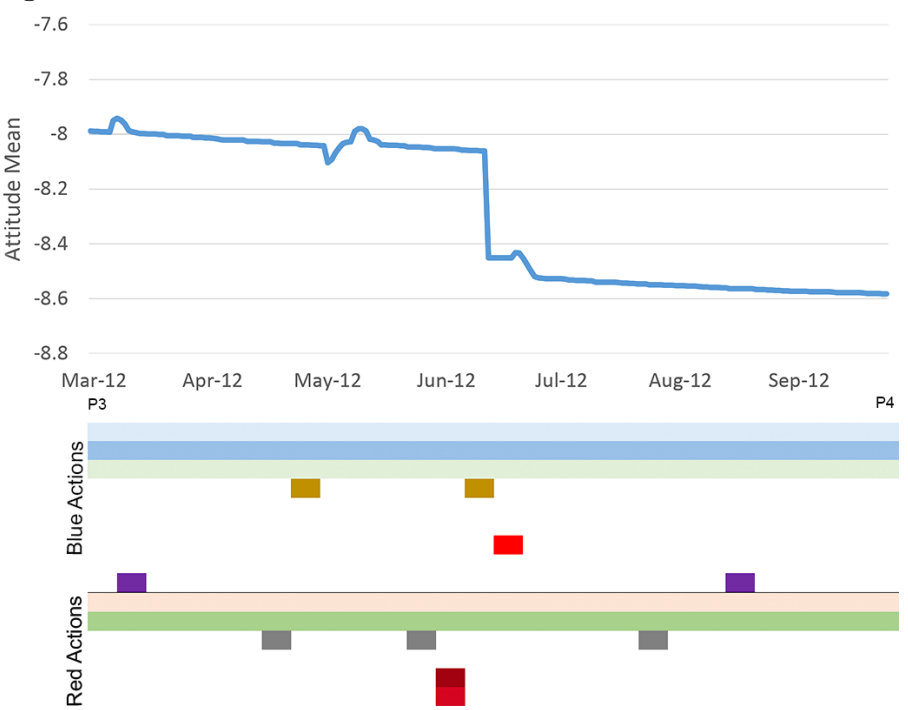

Fig. 5: Attitude mean toward Blue Force between P3 and P4

attitude decreasing toward Red Force at each IED (Improvised Explosive Device, gray blocs in the figure) and also that the first occurrence has the greatest impact since the population is surprised. Besides, we can notice that the impact of a suicide attack is much greater than other actions (mid-July 2011 and June 2013).

In figure 5, we can see that attitudes toward the Blue Force are also impacted by each of its actions. Moreover, we can notice that the curve greatly decreases when the suicide attack perpetuated by Reds occurs. This phenomenon is enabled by the mechanism of co-responsibility (see 4.2), the Blue Force is also responsible of these attacks, in a moderate way. Similarly, the attitude toward Blue Force decreases constantly since the background communication actions 
and patrols are not enough to counter their co-responsibility toward the pressure activity for Reds.

Role of co-responsibility To show the impact of the co-responsibility mechanism, we have performed a new simulation with $\rho=0$ (i.e. no co-responsibility). Since the initial values of attitudes toward Blue Force depend on $\rho$ during the initialization scenario, we had to re-calibrate the model parameters. Figure 6 shows the resulting attitude dynamics. As it was predictable, the attitude toward the Blue Force constantly increases as their only potentially negative actions (kinetic actions against the Red Force) are easily countered by communication toward the population or reconstruction actions. This is what was expected by the stakeholders when they decided to engage in Afghanistan.

The tendency of attitude toward Red Force remains the same since the coresponsibility only affects the Blue. We can notice that the simulated attitudes toward Reds is closer to opinion polls than in the first calibration. This is due to the fact that the red scenario is more simple (less action diversity).

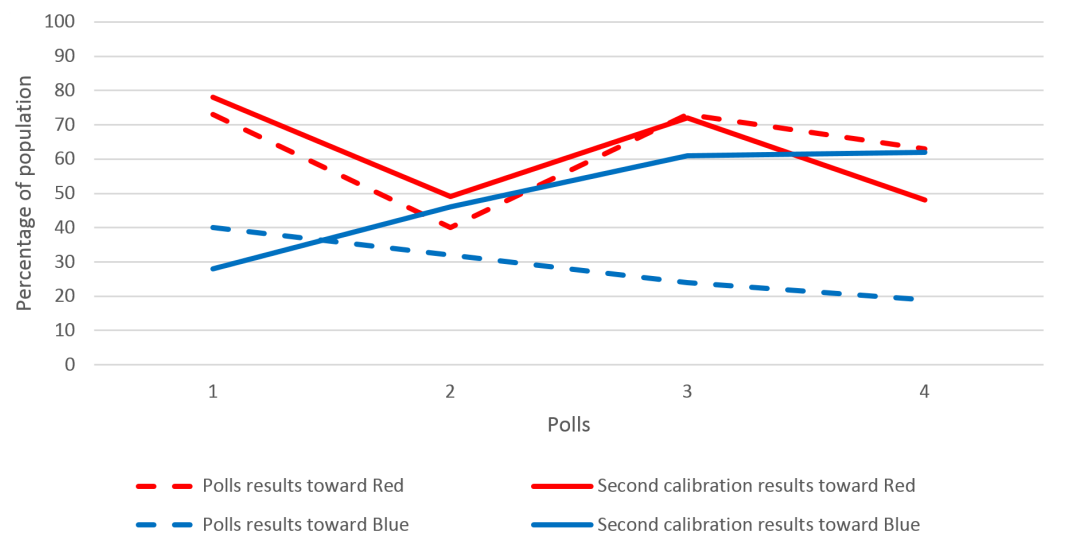

Fig. 6: Impact of co-responsibility

\section{Conclusion}

We have collected information on the opinion dynamics and the events during the involvement of French army in Afghan war. These information were analyzed and processed with the support of subject matter experts. Based on these field observations, we proposed a simulation model of attitude dynamics. This model embodies both cognitive and affective components in the formation of attitude and the diffusion of beliefs. While it was designed in the context of military operations, it can be applied to civilian use: the actors can represent any kind of active social object such as political parties, institutions, companies or brands. 
We also introduced a new concept of co-responsibility that reflects attitude behavior perceptible in conflict terrain. These components were aggregated through a new method that better understands the concept of attitude proposed by Fazio. We conducted a model calibration based on the collected data that showed encouraging results.

In future works, we intend to conduct deeper analysis of the field data to adapt the model and implement the simulation of different social groups that are present in this area. Moreover, we would like to implement a memory mechanism to let the agents "forget" some beliefs over time. This would be enabled for instance using the Peak-End mechanism of Kahneman [14]. Furthermore, we would like to add a behavioral component to enable population agents to express their attitudes through actions selection.

\section{References}

1. Icek Ajzen and Martin Fishbein. The influence of attitudes on behavior. In D. Albarrac, B. T. Johnson, and M. P. Zanna, editors, The handbook of attitudes, pages 173-221. Lawrence Erlbaum Associates Publishers, Mahwah, NJ, US, 2005.

2. Gordon W. Allport. Attitudes. 1935.

3. Kei-Léo Brousmiche, Jean-Daniel Kant, Nicolas Sabouret, François PrenotGuinard, and Stéphane Fournier. The role of emotions on communication and attitude dynamics: an agent-based approach. WCSS, 2014.

4. C. Castellano, S. Fortunato, and V. Loreto. Statistical physics of social dynamics. Reviews of modern physics, 81(2):591, 2009.

5. G. Deffuant, D. Neau, F. Amblard, and G. Weisbuch. Mixing beliefs among interacting agents. Advances in Complex Systems, 3(01n04):87-98, 2000.

6. Adrian Dimulescu and Jean-Louis Dessalles. Understanding narrative interest: Some evidence on the role of unexpectedness. In Proceedings of the 31st Annual Conference of the Cognitive Science Society, pages 1734-1739, 2009.

7. Alexis Drogoul and Jacques Ferber. Multi-agent simulation as a tool for modeling societies: Application to social differentiation in ant colonies. In Artificial Social Systems, pages 2-23. Springer, 1994.

8. EMA and ECPAD (Ministry of Defence). Ensemble vers l'autonomie. Cinq ans en Kapisa et Surobi http://webdocs.ecpad.fr.

9. Russell H. Fazio. Attitudes as object-evaluation associations of varying strength. Social Cognition, 25(5):603, 2007.

10. Nikolaus Hansen, Sibylle Mller, and Petros Koumoutsakos. Reducing the time complexity of the derandomized evolution strategy with covariance matrix adaptation (CMA-ES). Evolutionary Computation, 11(1):1-18, 2003.

11. Herbert Lionel Adolphus Hart. Punishment and responsibility: Essays in the philosophy of law. Oxford University Press, 2008.

12. Pahl-Wostl and Claudia Johannes Kottonau. Simulating political attitudes and voting behavior, October 2004.

13. E. E. Jones and V. A. Harris. The attribution of attitudes. Journal of Experimental Social Psychology, 3(1):1-24, 1967.

14. Daniel Kahneman, D. Kahneman, and A. Tversky. Experienced utility and objective happiness: A moment-based approach. The psychology of economic decisions, 1:187-208, 2003. 
15. H. Kelley. The processes of causal attribution. American psychologist, 28(2), 1973.

16. Stanley Milgram. The small world problem. Psychology today, 2(1):60-67, 1967.

17. A. Nowak, J. Szamrej, and B. Latane. From private attitude to public opinion: A dynamic theory of social impact. Psychological Review, 97(3):362, 1990.

18. M. J. Rosenberg and C. I. Hovland. Cognitive, affective, and behavioral components of attitudes. Attitude organization and change: An analysis of consistency among attitude components, 3:1-14, 1960.

19. Rupert Smith. The utility of force: the art of war in the modern world. Knopf, New York, 2007.

20. Diemo Urbig and Robin Malitz. Drifting to more extreme but balanced attitudes: Multidimensional attitudes and selective exposure. ESSA, Toulouse, 2007. 